\title{
Metastização óssea de carcinoma hepatocelular: a propósito de um caso clínico
}

\author{
Bone metastasis of hepatocellular carcinoma: a case report
}

\section{RESUMO}

0 carcinoma hepatocelular (CHC) é o tumor hepático mais frequente, com uma incidência crescente ao longo dos anos. A metastização extra-hepática após terapêutica loco-regional é pouco frequente, sendo o osso um local pouco afetado.

Os autores apresentam o caso de um doente de 72 anos, com antecedentes pessoais de CHC (controlado sob radiofrequência), que recorreu ao Serviço de Urgência por dor no membro superior direito com início após mau posicionamento. A radiografia mostrou fratura da extremidade distal do úmero, tendo realizado radiografia de controlo após 2 meses que apresentava ausência de osso no local onde tinha ocorrido a fratura, agora substituído por lesão expansiva. Após estudo complementar admitiu-se tratar-se de metástase óssea do CHC. 0 doente faleceu 1 mês após o diagnóstico.

0 caso apresentado alerta para a importância da exclusão de fraturas patológicas em doentes com antecedentes pessoais de neoplasia, o que, caso se verifique, poderá alterar a abordagem e prognóstico destes doentes.

\section{ABSTRACT}

Hepatocellular carcinoma (HCC) is the most frequent liver tumor, with an increasing incidence over the years. Extrahepatic metastasis after locoregional therapy is infrequent, with bone being a poorly affected site.

The authors present the case of a 72 year-old man, with a personal history of HCC (controlled under radiofrequency) who appealed to the Emergency Department for pain in the upper right limb after poor positioning. The radiograph showed fracture of the distal end of the humerus, and a control radiograph was performed after 2 months, which showed no bone at the site where the fracture had occurred, now replaced by an expansive lesion. After a complementary study it is admitted that bone metastasis of HCC is involved. The patient died 1 month after diagnosis.

The present case alerts to the importance of the exclusion of pathological fractures in patients with a personal history of neoplasia, which, if happens, could alter the approach and prognosis of these patients.

Palabras clave: carcinoma hepatocelular, metástasis, hueso.

Keywords: hepatocellular carcinoma, metastization, bone.

\section{CASO CLÍNICO}

Os autores apresentam o caso de um doente de 72 anos, com antecedentes de hepatite B crónica e carcinoma hepatocelular (CHC) (lesão única de $3 \mathrm{~cm}$, diagnosticada 1 ano antes e controlada sob radiofrequência) que recorreu ao Serviço de Urgência por dor no membro superior direito com início após mau posicionamento. A radiografia mostrou fratura da extremidade distal do úmero (Fig. 1), tendo sido colocada tala gessada.

Na radiografia de controlo após 2 meses apresentava ausência de osso no local onde tinha ocorrido a fratura (Fig. 2), agora substituído por lesão expansiva de etiologia a esclarecer, pelo que foi internado no Serviço de Medicina.
Figura 1. Radiografia do cotovelo direito que mostra fratura da extremidade distal do úmero

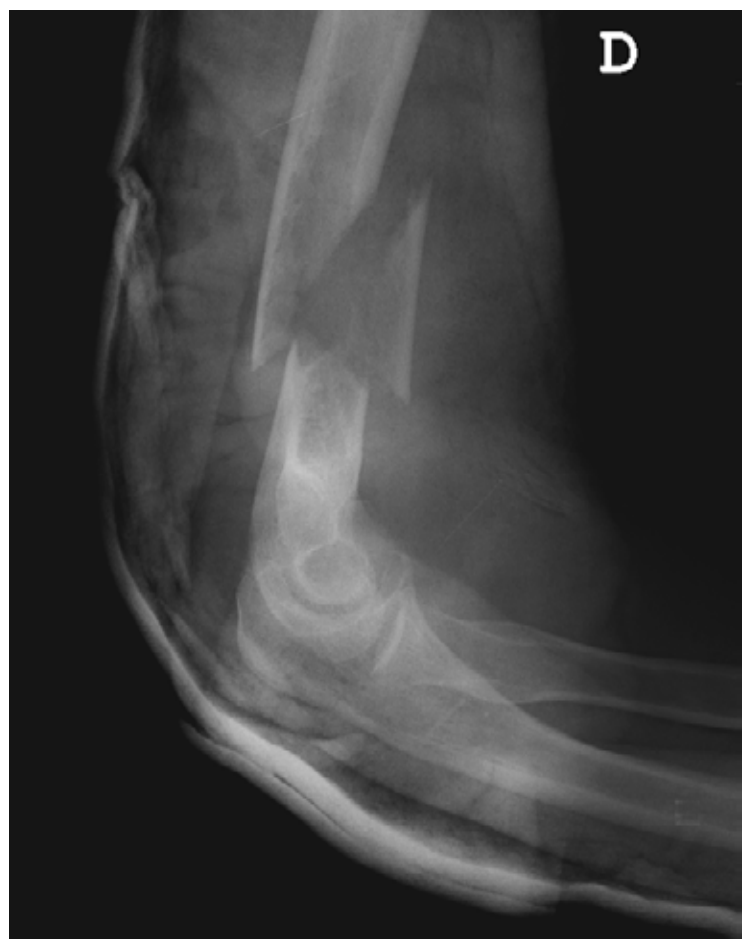

Figura 2. Radiografia do cotovelo direito que mostra ausência de osso na extremidade distal do úmero, substituído por lesão expansiva

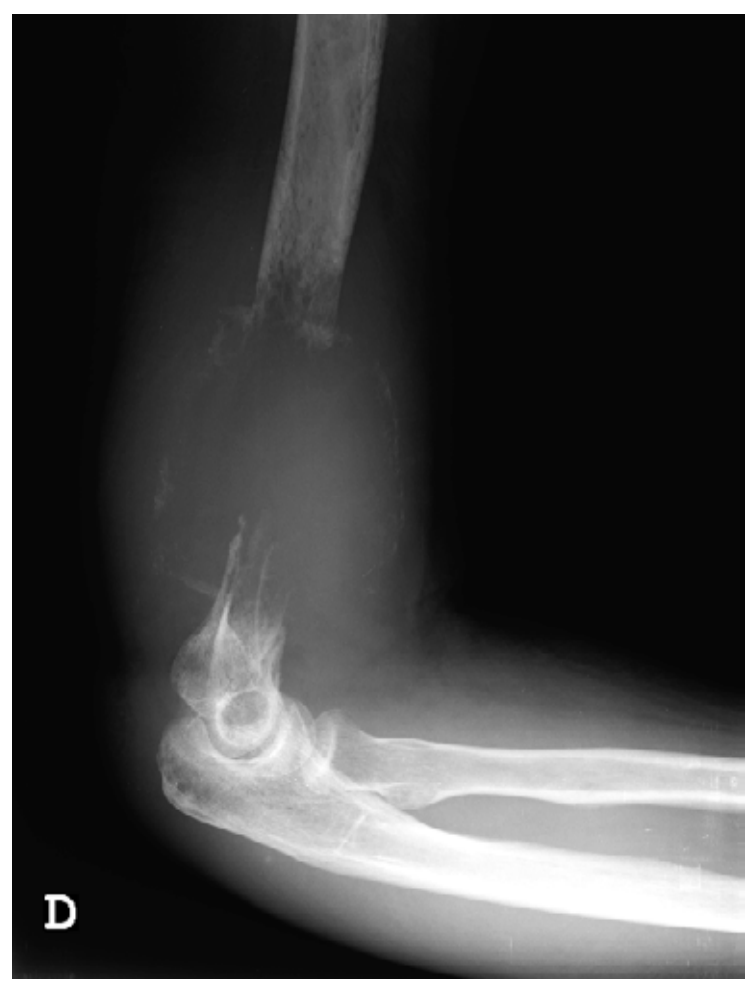


Ao exame objetivo apresentava deformação do membro afetado. Analiticamente acentuada elevação da alfa-fetoproteína (de 18 para $532 \mathrm{UI} / \mathrm{mL}$, com 9 meses de intervalo; normal $<5.5 \mathrm{Ul} / \mathrm{mL}$ ) e agravamento do padrão de citocolestase hepática. Realizou tomografia computorizada (TC) do cotovelo que mostrou no "úmero direito lesão lítica expansiva com cerca de 7.6x8.4x6.6 cm, sem plano de clivagem com os diferentes grupos musculares do antebraço". Posteriormente realizou cintigrafia óssea que, para além da lesão referida, mostrava "alterações sugestivas de lesões secundárias ao nível do ísquion esquerdo". TC toraco-abdomino-pélvica evidenciava "lesão hepática previamente conhecida, coexistindo outra lesão nodular no lobo esquerdo com cerca de $1.7 \mathrm{~cm}$ e múltiplas adenopatias na região hilar e nas cadeias do tronco celíaco, sem outras alterações valorizáveis". Foram excluídas outras neoplasias primárias, nomeadamente do pulmão, mama, próstata, rim e tiróide. 0 caso foi discutido em reunião multidisciplinar e, atendendo ao aumento recente e acentuado da alfa-fetoproteína, ao surgimento de nova lesão hepática e de múltiplas adenomegalias na região hilar hepática e nas cadeias do tronco celíaco (que previamente não existiam), admitiu-se tratar-se de CHC metastizado, pelo que não foi realizada biópsia óssea (para além do risco hemorrágico acrescido em virtude da trombocitopénia e distúrbios da coagulação que apresentava) e se decidiu por terapêutica paliativa.

Na ausência de alterações que sugerissem outro tumor primário e face à evidência de progressão do carcinoma hepatocelular (alterações analíticas e imagiológicas), considerou-se que a lesão expansiva no úmero direito se tratava de metástase do referido carcinoma, pelo que se prescindiu da biópsia (para além do elevado risco hemorrágico previamente referido) e não foram realizados exames adicionais, para além dos supra-referidos.
0 doente foi referenciado para Consulta de Oncologia, tendo falecido antes da sua realização (1 mês após o diagnóstico de metastização).

0 CHC é o tumor hepático mais frequente, com uma incidência crescente ao longo dos anos. ${ }^{1}$ A metastização extra-hepática após terapêutica loco-regional é pouco frequente (5-24\%), sendo 0 osso um local pouco afetado. ${ }^{2,3} \mathrm{~A}$ evidência de metastização está associada a pior prognóstico, ${ }^{4}$ tal como se constatou no caso descrito.

\section{BIBLIOGRAFÍA}

1. Ruiz-Morales JM, Dorantes-Heredia R, Chable-Montero F, Vazquez-Manjarrez S, Méndez-Sánchez N, Motola-Kuba D. Bone metastasis as the initial presentation of hepatocellular carcinoma. Two case reports and a literature review. Ann Hepatol. 2014 Nov-Dec; 13(6): 838-842

2. Senthilnathan S, Memon K, Lewandowski RJ, Kulik L, Mulcahy MF, Riaz A, et al. Extrahepatic metastases occur in a minority of hepatocellular carcinoma patients treated with locoregional therapies: analyzing patterns of progression in 285 patients. Hepatology. 2012 May; 55(5):1432-1442

3. Tonolini M, Solbiati L, lerace T, Kirn V, Croce F. Extrahepatic recurrence and second malignancies after treatment of hepatocellular carcinoma: spectrum of imaging findings. Radiol Med. 2002 Mar; 103(3):196-205

4. Schwartz JM, Carithers RL. Clinical features and diagnosis of primary hepatocellular carcinoma. UpToDate [consultado em dezembro 2016]. Disponível em:

https://www.uptodate.com/contents/clinical-features-and-diagnosis-of-primary-hepatocellular-carcinoma

\section{DIAGNÓSTICO}

Metastização óssea de carcinoma hepatocelular

\section{Sónia Martins, Ana Sanches}

Serviço de Medicina V. Centro Hospitalar do Médio Tejo (CHMT) 